\title{
O Modelo de Thirlwall com variações nas elasticidades ${ }^{1}$
}

\author{
Luciano Nakabashi ${ }^{2}$
}

\section{Resumo}

Fluxo de capitais é um importante elemento no crescimento econômico por diversos motivos. Um deles é porque ele alivia a restrição no balanço de pagamentos de acordo com a Lei de Thirlwall. A explicação é que pelo fato de ocorrer uma elevação no nível de importações quando há um crescimento da renda de uma determinada economia deverá ocorrer uma entrada de recursos via exportações ou fluxo de capitais para que seja possível o fechamento do balanço de pagamentos (BP). O foco do presente trabalho está na avaliação de como o fluxo de capitais afetou o crescimento da economia brasileira no período 1947-2000, considerando que as elasticidades-renda das importações mudam ao longo do tempo.

Palavras-chave: Elasticidade-renda (Economia); Fluxo de capitais; Desenvolvimento econômico; Brasil - Importações; Balanço de pagamentos - Restrição.

\section{Abstract}

\section{Thirlwall's Model with elasticities changes}

Capital flows are expected to be important in the process of economic growth for several reasons. One of them is their importance in relaxing the balance of payments constraints as envisaged by Thirlwall's law, which states that when economic growth takes place, the level of imports also grows. Consequently, there is an increase in the export level or in the volume of capital inflows as imposed by the balance of payment constraints. The goal of the present paper is to investigate how capital flows have stimulated the Brazilian economy by means of Thirlwall's model in the 19472000 period of time, taking into consideration that income elasticity of imports changes over time.

Key words: income elasticity of imports; capital flows; balance of payment constraint.

JEL E12; N16; O11; O54.

\section{Introdução}

O debate sobre os determinantes do crescimento econômico tem sido vigoroso. Existem os pesquisadores que enfatizam o lado da oferta e outros que exaltam elementos da demanda como essenciais no processo. Um breve e recente debate sobre a importância dos fatores da oferta e da demanda para explicar o desempenho econômico brasileiro foi feito por Barbosa (2006) e Oreiro (2006).

Considerando os economistas que enfatizam o papel da demanda, Kaldor (1988) tem um papel proeminente ao lançar as bases para um modelo formal onde

(1) Trabalho recebido em setembro de 2004 e aprovado em dezembro de 2006.

(2) Doutor em economia pelo Centro de Desenvolvimento e Planejamento Regional (Cedeplar)/ Universidade Federal de Minas Gerais (UFMG) e professor adjunto da Universidade Federal do Paraná (UFPR). Agradeço os comentários feitos por Gabriel Porcile e pelo parecerista anônimo. E-mail: <luciano.nakabashi@ufpr.br>. 
o crescimento da renda depende do desempenho da demanda efetiva. Atesoglu (2002, 1996) apresenta algumas evidências empíricas sobre a importância da demanda no crescimento de longo prazo da economia norte-americana e alemã, respectivamente.

Recentemente, a abordagem pós-keynesiana tem enfatizado o papel da demanda através da utilização do modelo desenvolvido por Thirlwall (1979), em que as restrições externas ao crescimento possuem um papel fundamental nesse processo e, portanto, a demanda externa é um dos principais motores do dinamismo econômico. Nesse modelo, as elasticidades-renda das importações e das exportações são elementos-chave para um bom desempenho econômico de longo prazo (McCombie; Roberts, 2002). Posteriormente, o modelo foi estendido por Thirlwall e Hussain (1982) para incluir fluxos de capital. Moreno-Brid (1998) traz avanços adicionais ao introduzir uma restrição que limita o crescimento do déficit em conta corrente como proporção da renda doméstica. O país tem que manter certa proporção entre as duas variáveis de modo a obter um crescimento sustentável no longo prazo. McCombie e Thirlwall (1997) fazem avanço similar no sentido de introduzir uma restrição para que a razão entre os déficites comerciais e a renda seja constante. No entanto, Barbosa-Filho (2002) mostra que essa condição não é suficiente para impedir uma elevação da dívida externa em níveis que não sejam sustentáveis, pois mesmo com uma razão constante entre déficit em conta corrente e renda doméstica, a trajetória do crescimento da dívida pode chegar a níveis que gerem crises de confiança de modo a tornar insustentável o padrão atual de crescimento. $\mathrm{O}$ autor inclui na análise, de forma explícita, o pagamento de juros e a dinâmica da dívida externa para contornar o problema.

Porcile e Curado (2002) desenvolvem um modelo estruturalista Norte-Sul, onde a existência de um hiato tecnológico que limita o crescimento do Sul acaba por gerar desequilíbrios no Balanço de Pagamentos (BP). Esses desequilíbrios limitam ainda mais o crescimento do Sul, pois o forçam a depender da entrada de capitais para a manutenção de uma taxa de crescimento que seja consistente com o pleno-emprego, o que, por sua vez, eleva os riscos de default com todas as consequiências negativas sobre o crescimento que tal processo induz.

As várias vertentes do modelo já foram testadas inúmeras vezes, inclusive para o caso brasileiro. Bértola et al. (2002) fazem uso do modelo mais simples e encontram uma relação de longo prazo entre o desempenho do PIB brasileiro, termos de troca e o crescimento da renda mundial, no período 1890-1973, favorecendo a Lei de Thirlwall. Na análise do período 1955-1998, Jayme Jr. (2001), utilizando o método de co-integração em séries temporais, encontra que há co-integração entre o crescimento das exportações e o crescimento econômico, o que indica a validade do modelo de Thirlwall. Nakabashi (2003) mostra que o modelo com fluxos de capital é compatível com o crescimento da economia 
brasileira para o período 1947-2000, enquanto o mesmo não é verdade para o modelo sem fluxos de capital (Lei de Thirlwall).

Porcile et al. (2003) e Barbosa-Filho (2004), por outro lado, empregam o modelo de Thirlwall para analisar questões de curto prazo. Os primeiros combinam o modelo de Thirlwall com o conceito minskyano de fragilidade financeira adaptado para uma economia aberta para analisar a conjuntura econômica latino-americana, enquanto o segundo tem como objetivo estudar o trade-off entre crescimento e taxa de câmbio. Seus resultados indicam que, no caso de se desejar elevar a taxa de crescimento da renda da economia brasileira em 1\%, seria necessária uma desvalorização cambial de $7 \%$ para se manter constante a razão saldo da balança comercial/PIB. Suas conclusões são de que a elasticidaderenda das importações do Brasil é muito elevada e as elasticidades-preço das importações e exportações baixas, prejudicando o alcance de um elevado crescimento sustentável da economia.

É preciso, porém, atentar para a questão da relação de causalidade entre as elasticidades-renda das importações e exportações e a taxa de crescimento da renda. Krugman (1988) sugere que são as elasticidades-renda das importações e das exportações que se ajustam às taxas de crescimento dos países (45-degree rule). Para Krugman, não há muitas razões para se supor que as restrições externas causem impactos sobre a taxa de crescimento da renda, no longo prazo.

It just seems fundamentally implausible that over stretches of decades balance of payments problems could be preventing long term growth, especially for relatively closed economies like the US in the 1950s and 1960s. Furthermore, we all know that differences in growth rates among countries are primarily determined by differences in the rate of growth of total factor productivity ... (Krugman, 1988, p. 12).

No entanto, Thirlwall também tem boas razões para suspeitar que o desempenho das importações e exportações possa ter um papel crucial no crescimento de uma determinada economia e/ou como uma restrição ao crescimento, pois déficits em conta corrente, restringindo o crescimento econômico, parecem ser um problema de grande importância por, pelo menos, três motivos: (1) efeitos sobre os setores diretamente afetados pelo aumento das importações e/ou queda das exportações; (2) nenhum país pode crescer mais rápido que a taxa de crescimento com equilíbrio no $\mathrm{BP}$, pelo menos no longo prazo, pois um déficit crescente na conta corrente financiado através da conta capital aumentaria o risco de desvalorizações cambiais até o ponto em que não valeria mais a pena investir em tal região ou país e este teria que se ajustar à nova situação através de medidas recessivas (espontâneas ou forçadas); e (3) um déficit crescente em conta corrente levaria o país ou região a praticar taxas de juros mais elevadas para atrair fluxos de capital, estimulando a parte financeira em prejuízo da parte real da economia. 
A essência do modelo é que o país deve manter o saldo do BP em equilíbrio, no longo prazo, pois um país não pode se endividar continuamente sem nunca ter que pagar sua dívida. ${ }^{3}$ Como o montante de importações depende da demanda do país, que por sua vez é uma função da renda doméstica, caso o país tenha um déficit na balança comercial chegará um momento em que ele deverá reduzir seu crescimento, elevar o montante de bens e serviços exportados para inverter o déficit ou reduzir a elasticidade-renda das importações para pagar a dívida existente. Esta última não tem sido enfatizada na literatura teórica ou empírica principalmente pelo fato de o modelo de Thirlwall (1979) e de suas derivações terem, por suposição, uma elasticidade-renda das importações constante, sendo este um dos principais focos do presente estudo.

O objetivo do presente trabalho é tentar responder, com base no modelo desenvolvido por Thirlwall e Hussain (1982), para incluir fluxos de capital, se as elasticidades-renda das importações são de fato constantes. Se esse não for o caso, as restrições externas não são tão severas quanto preconizadas pela Lei de Thirlwall, já que as elasticidades se ajustam a alterações no cenário econômico internacional de modo a suavizar o crescimento da economia. Portanto, a explicação teórica de restrição ao crescimento baseada no modelo desenvolvido por Thirlwall e Hussain (1982) é apenas parcialmente compatível com as séries de dados. Adicionalmente, ao comparar o comportamento das duas elasticidades (real da economia e com restrição no balanço de pagamentos), pode-se notar que a proximidade das duas é maior em certos momentos históricos, indicando que o modelo de Thirlwall e Hussain (1982) é mais adequado para explicar o desempenho da economia brasileira em determinados momentos em relação a outros, apesar de alguns estudos mostrarem que o modelo se ajusta bem aos dados quando se considera todo o período.

Além desta introdução, o estudo está dividido em: 1) apresentação do modelo de Thirlwall e Hussain (1982); 2) metodologia e dados; e 3) apresentação dos resultados empíricos juntamente com uma análise histórica.

\section{0 modelo de crescimento de Thirlwall e Hussain}

Seguindo o modelo desenvolvido por Thirlwall e Hussain (1982), com a inclusão de fluxos de capitais, nele se começa com a suposição de equilíbrio no $\mathrm{BP}$ (e possível desequilíbrio na balança comercial quando $\mathrm{F} \neq 0$ ) medido em unidades de moeda doméstica:

$$
P_{d} X+F=P_{f} M E
$$

em que $\mathrm{X}$ representa o volume de exportações, F o valor nominal do fluxo de capitais em moeda doméstica, embora no presente trabalho tenhamos utilizado

(3) Na verdade, um país até pode se endividar continuamente sem nunca ter que pagar a dívida acumulada, mas essa situação só é sustentável caso haja um crescimento da economia de modo a não elevar a relação dívida/PIB além de um determinado limite. 
a soma dos fluxos de capital e serviços pelo fato de este ser de extrema importância no caso brasileiro, $\mathrm{P}_{\mathrm{d}}$ é o preço das exportações, em moeda doméstica, $\mathrm{M}$ é a quantidade de importações, $\mathrm{P}_{\mathrm{f}}$ é o preço das importações, em moeda estrangeira, e E é a taxa de câmbio nominal (preço doméstico da moeda estrangeira).

Transformando em taxas de crescimento obtemos:

$$
\theta\left(p_{d}+x\right)+(1-\theta) f=p_{f}+m+e
$$

em que as letras minúsculas representam as taxas de crescimento das variáveis, $\theta$ e $(1-\theta)$ são as parcelas das exportações e dos fluxos de capitais somados aos da conta de serviços no total da receita obtidas pelo setor externo, ou seja, qual a parcela do total de importações é paga pelas exportações e qual é pela entrada de capitais e conta de serviços.

A quantidade demandada de importações pode ser especificada como uma função multiplicativa dos preços das importações (medidas em unidades monetárias domésticas), dos preços dos seus substitutos e da renda doméstica:

$$
M=\alpha\left(\frac{P_{f} E}{P_{d}}\right)^{\varphi} Y^{\pi}
$$

em que $\alpha$ é uma constante, $\varphi$ é a elasticidade-preço da demanda por importações $(\varphi<0)$, Y é a renda doméstica e $\pi$ é a elasticidade-renda da demanda por importações $(\pi>0)$.

Em taxas de crescimento temos:

$$
m=\varphi\left(p_{f}+e-p_{d}\right)+\pi y
$$

A quantidade demandada de exportações também pode ser representada por uma função multiplicativa dos preços das exportações, do preço das mercadorias que competem com as exportações (medidas em unidades de moeda doméstica) e do nível da renda mundial:

$$
X=\beta\left(\frac{P_{d}}{P_{f} E}\right)^{\eta} Z^{\varepsilon}
$$

em que $\beta$ é uma constante, $\eta$ é a elasticidade-preço da demanda por exportações $(\eta<0), Z$ é a renda mundial e $\varepsilon$ é a elasticidade-renda da demanda por exportações $(\varepsilon>0)$. Transformando em taxas de crescimento, temos:

$$
x=\eta\left(p_{d}-p_{f}-e\right)+\varepsilon z
$$

Substituindo as equações (4) e (6) em (2) e isolando y do lado esquerdo da equação, encontramos:

$$
y_{b}=\frac{(\theta \eta+\varphi+1)\left(p_{d}-p_{f}-e\right)+\theta \varepsilon z+(1-\theta)\left(f-p_{d}\right)}{\pi}
$$


Nessa equação, a nossa variável dependente $\left(\mathrm{y}_{\mathrm{b}}\right)$ é a taxa de crescimento com equilíbrio no BP. Na equação (7), ainda podemos considerar que $\varepsilon z=x$, onde $\mathrm{x}$ é a taxa de crescimento das exportações.

\section{Metodologia e dados}

Neste trabalho será testado o modelo de Thirlwall e Hussain (1982) para o caso brasileiro com o objetivo de se analisar sua capacidade explicativa e ver se ele contribui para um melhor entendimento do papel das restrições externas sobre o crescimento. No presente estudo não foi realizado o teste tradicional proposto por Thirlwall e Hussain (1982), que seria o de verificar se a taxa de crescimento com restrição no BP, apresentada na equação (7), é estatisticamente igual à taxa real de crescimento da economia.

A metodologia utilizada foi a de calcular a elasticidade-renda das importações de acordo com a Lei de Thirlwall $(\pi$ '), ou seja, no caso em que o crescimento do país realmente está sendo restringido pela performance no BP.

$$
y_{b}=a j+b x+c f_{r}
$$

onde, $\mathrm{y}_{\mathrm{b}}$ é a taxa de crescimento da renda real, $\mathrm{x}$ é a taxa de crescimento das exportações $(x=\varepsilon z), \mathrm{f}_{\mathrm{r}}$ é a taxa real de crescimento dos fluxos de capital e serviços $\quad\left(f_{r}=f-p_{d}\right), \quad a=(\theta \eta+\varphi+1) / \pi^{\prime}, \quad b=\theta / \pi^{\prime}, \quad c=(1-\theta) / \pi^{\prime}$, $j=\left(p_{d}-p_{f}-e\right)$. Se soubermos qual parcela da entrada de divisas é composta pela exportação de bens e serviços $(\theta)$, podemos achar $\pi$ ' (elasticidade-renda das importações, caso a taxa de crescimento da economia esteja restrita ao desempenho do BP) através do coeficiente $b$.

Em seguida, $\pi$ ' foi comparada com a elasticidade-renda das importações real da economia $\left(\pi^{*}\right)$ calculada a partir do comportamento das importações como resposta ao crescimento da renda no Brasil. Para isso, foram feitas regressões a partir da equação (4), que também pode ser expressa como:

$$
m=\varphi j+\pi^{*} y
$$

em que $m$ é a taxa de crescimento das importações, $\varphi$ é a elasticidadecâmbio real das importações e $\pi^{*}$ é a elasticidade-renda das importações da economia. A partir dessas estimações, poderíamos calcular se $\pi$ ' é estatisticamente igual a $\pi^{*}$. No entanto, considerando que as elasticidades mudam através do tempo, foram feitas regressões por Mínimos Quadrados Ordinários (MQO) para vários subperíodos diferentes, de quatro anos cada $(n=4)$, totalizando 50 regressões, uma para cada elasticidade dos diferentes subperíodos. ${ }^{4}$ Desse modo, podemos verificar qual o comportamento das elasticidades e se, ao longo do tempo, elas apresentam as mesmas tendências ou se são opostas. É importante

(4) Por exemplo, a primeira regressão é feita para os anos 1948-1951, a segunda para 1949-1952 e assim por diante. 
notar que $\theta$ também varia para cada subperíodo e, portanto, existem $50 \theta$ diferentes. No entanto, como cada uma das 50 regressões possui uma amostra de tamanho 4, não é aconselhável a utilização de inferência estatística para realização de testes de hipóteses devido à elevada variância. O problema é que se não podemos testar hipóteses sobre os parâmetros, não podemos dizer se eles, portanto as elasticidades, são estatisticamente diferentes nos diversos períodos de análise, pois como os estimadores são estocásticos, eles devem variar de amostra para amostra mesmo quando os parâmetros populacionais são os mesmos.

Com o objetivo de contornar tal problema, também foram feitas regressões pelo método MQO utilizando variáveis dummies interativas em cada uma das regressões anteriores. No caso da equação (8), temos:

$$
y_{b}=a j+b x+c f_{r}+d D x
$$

onde D é uma variável dummy para cada um dos subperíodos. No entanto, diferentemente dos períodos das regressões anteriores, a primeira dummy é igual a 1 nas primeiras quatro observações (1948-1951) e zero nas demais, a segunda é igual a 1 nas próximas 4 observações (1952-1955) e zero nas demais, e assim por diante. Caso os períodos fossem separados como anteriormente, teríamos mais parâmetros do que o tamanho da amostra, tornando-se impraticável a estimação dos mesmos. Assim, o número de elasticidades cai para apenas 13. ${ }^{5}$ Com 53 observações e 38 graus de liberdade podemos, agora, utilizar testes estatísticos para verificar se as elasticidades são estatisticamente diferentes. Com o emprego das variáveis dummies na equação (9), temos:

$$
m=\varphi j+\pi^{*} y+v D y
$$

A análise será realizada para o Brasil entre 1947-2000. Os dados da renda, das importações, das exportações, da movimentação da conta de capitais e serviços, da taxa de câmbio e do índice de preços do Brasil foram retirados do Boletim do Banco Central e IBGE e estão em reais. A série de índices de preços dos Estados Unidos foi retirada do Bureau of Labor Statistics Washington. As séries foram deflacionadas (preços de 1995) e o software utilizado na análise de regressão foi Stata 8.1 .

\section{Análise empírica}

Na Figura 1 estão apresentados os resultados das regressões baseadas nas equações (8) e (9), sendo assim, os coeficientes de 100 diferentes regressões. $^{6}$ Podemos ver que as elasticidades estão próximas em alguns subperíodos do

(5) $\mathrm{Na}$ verdade, foram estimadas apenas 12 elasticidades, pois caso as 13 fossem utilizadas na regressão, cairíamos na armadilha da variável dummy, ou seja, as exportações seriam uma combinação linear exata das variáveis dummies. A primeira dummy não foi incluída, assim as demais são a diferença em relação à primeira. Na última dummy, os últimos 5 anos são iguais a 1.

(6) 50 para estimar as elasticidades reais de economia e 50 para estimar as elasticidades com restrição no balanço de pagamentos.

Economia e Sociedade, Campinas, v. 16, n. 1 (29), p. 93-110, abr. 2007. 
intervalo 1947-2000, mas distantes em outros. Do início do período de análise até o começo dos anos 1980, podemos dizer que o modelo de Thirlwall explica bem o comportamento da economia brasileira, com algumas pequenas exceções. ${ }^{7}$ Nos anos 1980, a relação entre as duas variáveis se perde ou se torna até mesmo negativa. Isso se deve às grandes alterações pelas quais a economia passou no período, como a grande redução do crescimento econômico, a brusca alteração no fluxo de capitais e a necessidade de mudança no desempenho da balança comercial para gerar superávits devido à nova situação da economia mundial.

Analisando o coeficiente de correlação entre as duas variáveis, do começo do período até o início dos anos 1980, ele é de 0,36 e, retirando quatro subperíodos entre 1973 e $1979,{ }^{8}$ onde as duas elasticidades tendem a caminhar em direção oposta, o coeficiente de correlação se eleva para 0,65. Já para os anos 1980, ela é de -0,37, o que acaba prejudicando a correlação quando analisamos o período como um todo, que é de -0,12. Nos anos 1990, como podemos ver pela Figura 1, elas voltam a ficar bem próximas.

Figura 1

Elasticidades-renda das importações real e com restrição no balanço de pagamentos

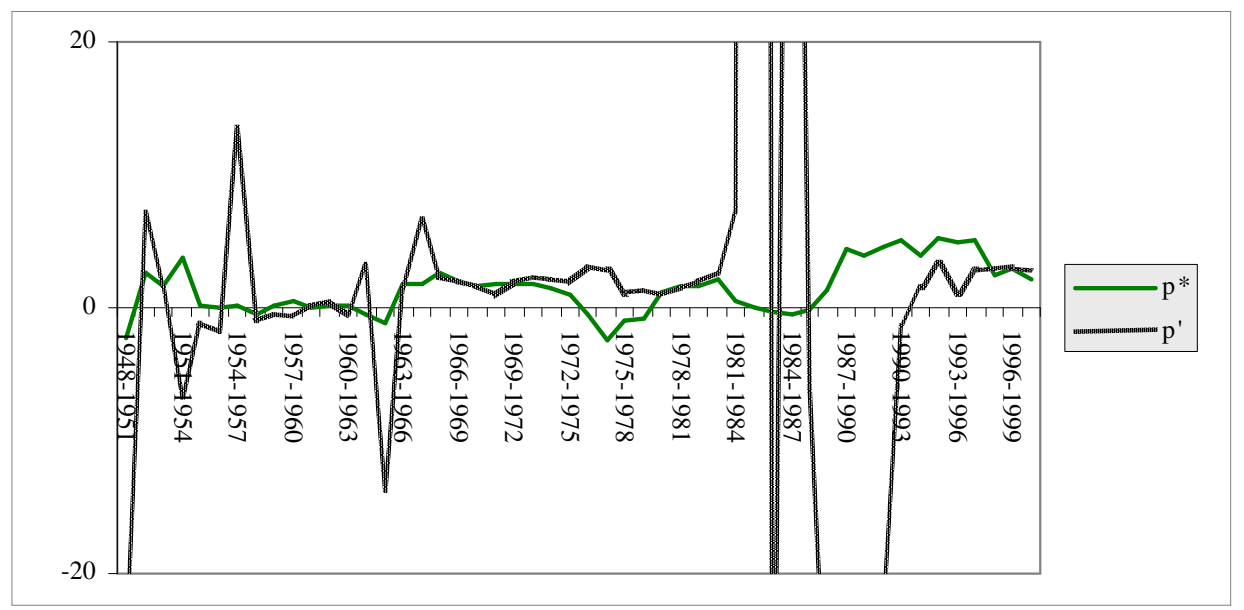

É interessante notar que a grande diferença no comportamento das duas elasticidades nos anos 1980 pode acabar prejudicando os resultados da aplicação do modelo de Thirlwall no Brasil, quando consideramos o período como um todo. Assim mesmo, estudos empíricos mostram evidências de que o modelo se aplica para todo o período, como Jayme Jr. (2001) e Bértola et al. (2002).

A Figura 1 nos traz evidências de que as elasticidades, de fato, variam. No entanto, como mencionado anteriormente, não podemos afirmar que elas são

(7) Entre os subperíodos 1948-1951 e 1980-1983 temos 33 diferentes subperíodos. Dentre eles, apenas em 4 temos uma grande diferença entre as duas elasticidades: 1948-1951, 1951-1954, 1954-1957, 1962-1965, mas nada comparado com os anos 1980.

(8) Os subperíodos 1973-1976, 1974-1977, 1975-1978 e 1976-1979. 
estatisticamente diferentes devido à baixa confiabilidade dos resultados pelo reduzido tamanho da amostra. Assim, torna-se conveniente a análise de regressão utilizando a amostra de todo o período em questão, fazendo uso de variáveis dummies para estimar as elasticidades para cada subperíodo, ou seja, regressões utilizando as equações (10) e (11). Ao realizar a regressão utilizando a especificação da última equação citada, nenhum problema sério foi encontrado em relação à heterocedasticidade pelo teste de Breusch-Pagan/Cook-Weisberg, à multicolinearidade, utilizando o Fator de Inflação da Variância (FIV) e à autocorrelação, pelo teste de Durbin-Watson. Porém, utilizando o teste do Stata para normalidade dos resíduos, sendo este baseado em D'Agostine, Belanger e D'Agostine Jr. (1990 apud Park, 2003), ${ }^{9}$ os resultados evidenciam uma nãonormalidade dos resíduos. ${ }^{10}$ Assim, se utilizou do método iterativo dos mínimos quadrados com redistribuição de pesos (Iteratively Reweighted Least Squares). Esse método consiste em proceder a regressão por MQO e desconsiderar os valores residuais muito elevados. Posteriormente, processo iterativo começa com pesos atribuídos a cada resíduo de modo que os que possuem maiores valores recebem os menores pesos. $\mathrm{O}$ processo iterativo termina quando a maior variação de um peso para o outro na mudança de iteração não ultrapassa um determinado valor mínimo. Esse método é indicado para correção de erros não normais causados por variáveis independentes com elevada leverage ${ }^{11}$ que levam à presença de outliers. Uma análise dos dados revela que temos esse problema tanto na taxa de crescimento das exportações quanto do fluxo de capitais, levando à presença de outliers:

Figura 2

Resíduos da regressão baseada na equação (11)

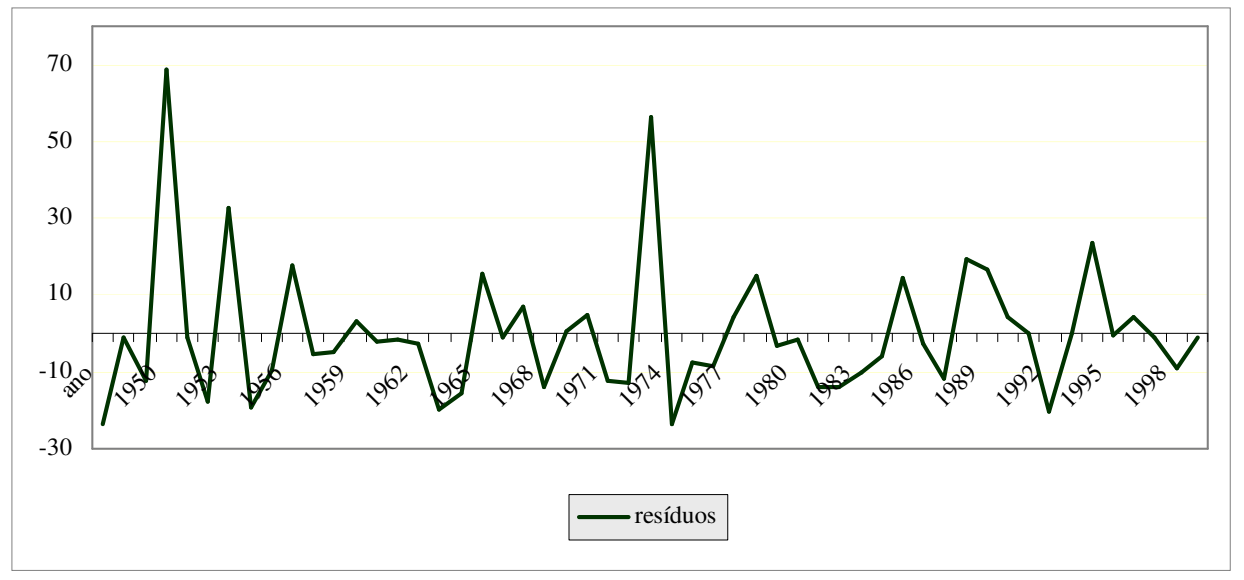

(9) O teste é construído através de uma medida de assimetria e outra de curtose da distribuição dos resíduos.

(10) Os resultados se encontram no apêndice.

(11) Variáveis com elevada leverage possuem observações com grandes distâncias do valor médio. 
No caso da regressão baseada na equação (10), também não foram encontrados problemas sérios de heterocedasticidade e multicolinearidade. ${ }^{12}$ Uma estimativa do teste de Durbin-Watson de 1,46 pode ser um indício de autocorrelação. No entanto, a regressão utilizando o método Cochrane-Orcutt $\mathrm{AR}(1)$ não apresentou alterações significativas nos resultados, sugerindo que o problema não é sério. Novamente, o teste para normalidade rejeita a hipótese nula devido à presença de assimetria na distribuição dos resíduos. Desse modo, se utilizou o método iterativo dos mínimos quadrados com redistribuição de pesos para eliminar problemas de outliers que levam à não-normalidade dos resíduos, embora, nesse caso, o problema não seja tão grave, como podemos ver na Figura 3.

Figura 3

Resíduos da regressão baseada na equação (10)

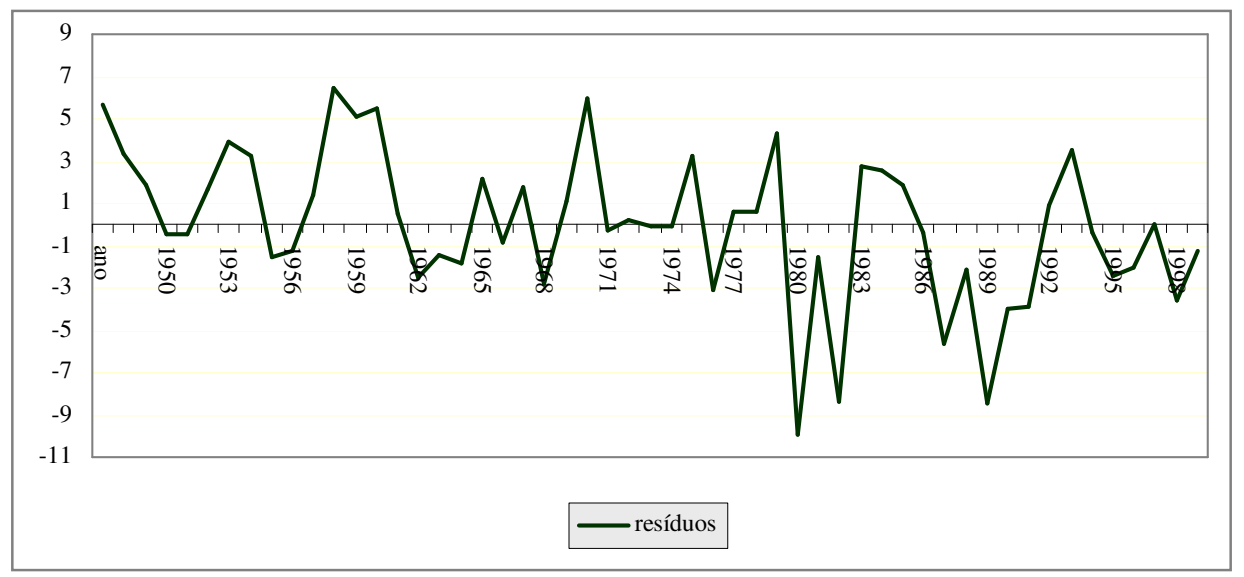

As elasticidades encontradas com o método de regressão acima mencionado para as elasticidades-renda das importações real $\left(\pi^{*}\right)$ e com restrição no balanço de pagamentos $\left(\pi^{\prime}\right)$ da economia brasileira se encontram na Figura 4 . O padrão de comportamento das elasticidades é diferente quando comparamos com o da Figura 1. No entanto, algumas semelhanças são notáveis, como a proximidade das duas do começo do período até o começo dos anos 1980, com exceção, em ambos os casos, no início, onde $\pi$ ' possui um valor muito baixo. Nos anos 1980 ocorre um distanciamento entre as duas e, nos 1990, uma tendência à aproximação. A diferença é que, no final do período, $\pi$ ' sofre uma grande redução no presente caso, enquanto no interior a tendência é de permanecer próximo de $\pi^{*}$. Assim, novamente fica registrada a observação de que o modelo desenvolvido por Thirlwall e Hussain (1982) explica melhor o desempenho econômico em certos momentos em relação a outros, apesar da possibilidade de ele permanecer válido no período como um todo.

(12) Um grau razoável de multicolinearidade foi encontrado entre as exportações e as variáveis dummies, mas isso seria de esperar por estas serem interativas com as primeiras. Todos os resultados estão no Apêndice. 
Figura 4

Elasticidades-renda das importações real e com restrição no balanço de pagamentos - dummies

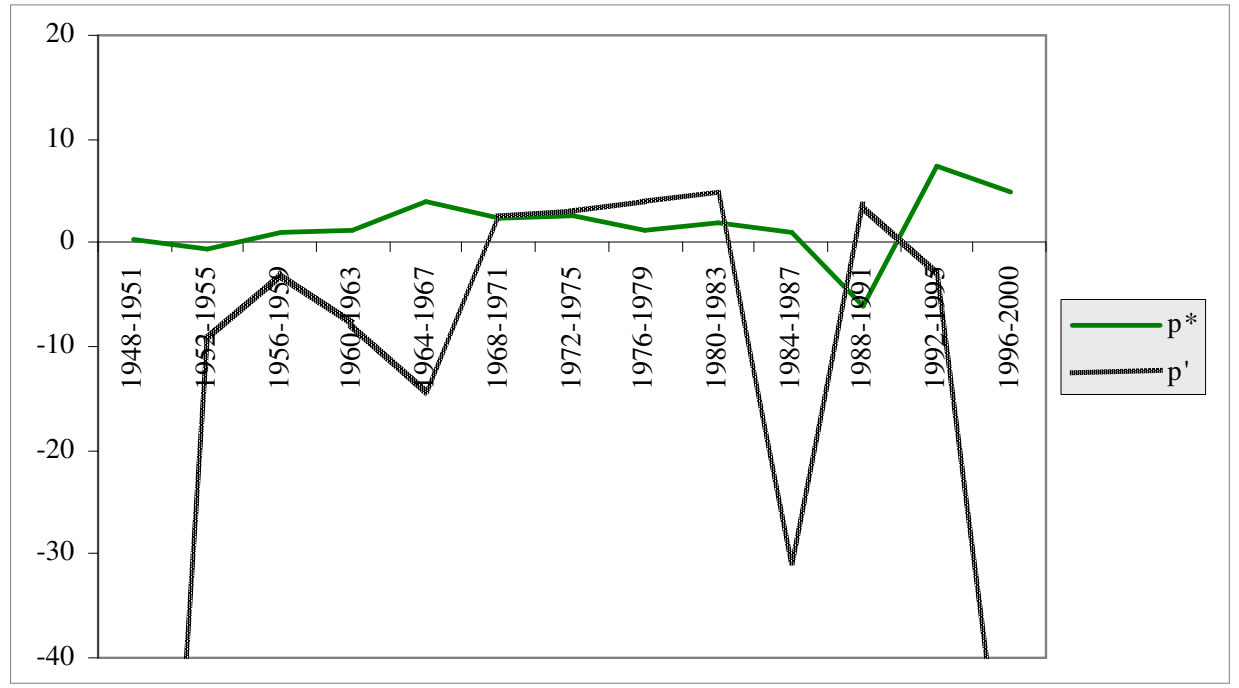

O principal ponto de interesse no presente estudo é verificar se as elasticidades-renda das importações, de fato, variam ao longo do tempo. Assim, precisamos fazer testes de hipótese para verificar se os coeficientes das variáveis dummies são estatisticamente diferentes. Utilizando testes $\mathrm{F}$ para verificar se os coeficientes das variáveis dummies, ${ }^{13}$ portanto, as elasticidades, são diferentes nos diversos subperíodos, chegamos aos seguintes resultados para $\pi^{*}$ (Tabela 1).

Para saber se a elasticidade do primeiro período é diferente da elasticidade dos demais foi utilizado o teste $\mathrm{F}$ para verificar se o coeficiente de cada variável dummy dos outros períodos é igual a zero, pois como o primeiro é o que serve de base, se algum outro coeficiente for estatisticamente igual a zero, isso implica que não houve alteração em relação à elasticidade do primeiro período.

Analisando os resultados da Tabela 1 , vemos que há evidências da existência de, pelo menos, quatro elasticidades distintas para o período como um todo. A primeira seria para os quatro primeiros períodos, uma segunda para os três subseqüentes, uma terceira para o décimo primeiro e mais uma para o décimo segundo período. Adicionalmente, há uma alternância das elasticidades. Por exemplo, a elasticidade dos quatro primeiro períodos parece voltar a ser a mesma nos períodos 8, 9 e 10. No entanto, olhando para os testes $F$ do coeficiente do segundo período em relação aos citados, pode ser que sua elasticidade seja distinta das dos demais. Esse seria o caso se estivéssemos dispostos a aceitar um nível de significância um pouco maior do que $10 \%$. A elasticidade do período 13 parece ser a mesma dos períodos 5 a 7 .

(13) Nas Tabelas 1 e 2, o coeficiente da segunda variável dummy é representado por d.2, da terceira por d.3, e assim por diante.

Economia e Sociedade, Campinas, v. 16, n. 1 (29), p. 93-110, abr. 2007. 
Tabela 1

Resultados de testes F para verificar se há diferença nas elasticidades reais $\left(\pi^{*}\right)$

\begin{tabular}{|c|c|c|c|c|c|c|c|c|c|c|c|c|c|}
\hline & d.1 & d.2 & d.3 & d. 4 & d. 5 & d.6 & d.7 & d. 8 & d. 9 & d.10 & d.11 & & d.13 \\
\hline d. 3 & & & - & 0.02 & $3.66^{*}$ & 2.55 & 2.80 & 0.05 & 0.56 & 0.01 & $6.15^{* *}$ & $15.41^{* * * *}$ & 2.69 \\
\hline d. 4 & & & & - & $3.08^{*}$ & 1.61 & 1.81 & 0.00 & 0.34 & 0.04 & $6.51^{* *}$ & $13.25^{* * *}$ & 2.40 \\
\hline d. 6 & & & & & & - & 0.01 & 1.39 & 0.16 & 1.87 & $9.25^{* * *}$ & $9.62^{* * *}$ & 1.06 \\
\hline d.7 & & & & & & & - & 1.56 & 0.21 & 2.02 & $9.55^{* * * *}$ & $9.23^{* * *}$ & 1.00 \\
\hline d. 8 & & & & & & & & - & 0.27 & 0.07 & $6.59^{* *}$ & $12.90^{* * *}$ & 2.31 \\
\hline d.9 & & & & & & & & & - & 0.52 & $7.56^{* * * *}$ & $8.56^{* * * *}$ & 1.28 \\
\hline d. 12 & & & & & & & & & & & & - & 1.04 \\
\hline d.13 & & & & & & & & & & & & & - \\
\hline
\end{tabular}

Notas: Testes F possuem 1 e 38 graus de liberdade no numerador e denominador, respectivamente. *, **, *** representam que os coeficientes estimados das variáveis dummies são estatisticamente diferentes ao nível de $10 \%, 5 \%$ e $1 \%$, respectivamente. Os valores nas células são as estimativas do teste $\mathrm{F}$.

Tabela 2

Resultados de testes F para verificar se há diferença nas elasticidades com restrição $(\pi$ ')

\begin{tabular}{|c|c|c|c|c|c|c|c|c|c|c|c|c|c|}
\hline & $\begin{array}{ll}\text { d. } \\
\end{array}$ & d.2 & d.3 & d.4 & d.5 & d.6 & d.7 & d.8 & d.9 & d.10 & d.11 & $\begin{array}{l}\text { d. } 12 \\
\end{array}$ & d.13 \\
\hline d. 3 & & & - & 0.65 & 0.69 & $5.66^{* *}$ & $5.78^{* *}$ & $3.52^{*}$ & $5.30^{* *}$ & 1.11 & $12.07^{* * *}$ & 0.01 & 1.32 \\
\hline d. 4 & & & & - & 0.05 & $4.78^{* *}$ & $5.12^{* *}$ & 2.13 & $4.05^{*}$ & 0.07 & $9.26^{* * *}$ & 0.47 & 0.27 \\
\hline d. 6 & & & & & & - & 0.47 & 0.29 & 0.14 & $4.32^{* *}$ & 1.76 & $6.13^{* *}$ & 1.94 \\
\hline d.7 & & & & & & & - & 0.01 & 0.07 & $5.09^{* *}$ & $4.07^{*}$ & $5.37^{* *}$ & 1.23 \\
\hline d. 8 & & & & & & & & - & 0.05 & 1.78 & $2.92^{*}$ & $3.37^{*}$ & 0.63 \\
\hline d.9 & & & & & & & & & - & $3.67^{*}$ & $2.91^{*}$ & $4.95^{* *}$ & 1.27 \\
\hline d. 12 & & & & & & & & & & & & - & 1.08 \\
\hline d.13 & & & & & & & & & & & & & \\
\hline
\end{tabular}

Notas: Testes $\mathrm{F}$ possuem 1 e 39 graus de liberdade no numerador e denominador, respectivamente. *, **, *** representam que os coeficientes estimados das variávei dummies são estatisticamente diferentes ao nível de $10 \%, 5 \%$ e $1 \%$, respectivamente. Os valores nas células são as estimativas do teste F. 
Seguindo a mesma linha de raciocínio, mas empregando os testes $\mathrm{F}$ nos coeficientes das variáveis dummies da equação (10) para testar se as elasticidades com restrição no BP $\left.(\pi)^{\prime}\right)$ variam.

De acordo com a Tabela 2, podemos ver que $\pi$ também varia. Comparando com os resultados da Tabela 1, o padrão de mudança é semelhante. A elasticidade com restrição no BP parece ser a mesma nos cinco primeiros períodos, com uma alteração nos próximos dois. Ela parece retornar aos valores dos primeiros cinco períodos no oitavo, décimo e nos dois últimos. A elasticidade do nono período parece retornar ao valor da elasticidade do sexto e sétimo, enquanto $\pi$ ' do décimo primeiro parece ser diferente de todos os outros. Desse modo, apesar de o padrão ser semelhante ao da Tabela 1, a elasticidade com restrição parece tomar apenas três diferentes valores no período como um todo. Na Tabela 3, podemos analisar com mais facilidade o padrão de mudança de $\pi^{*}$ e $\pi$ '. Nesta, a bola representa um mesmo padrão de alteração para as duas elasticidades, ou seja, as duas sofreram uma mudança estatisticamente significativa (ao nível de $10 \%$ ) ou ambas não sofreram alteração de um período para outro, enquanto o asterisco significa um diferente padrão de mudança, indicando que uma teve alteração estatisticamente significativa da elasticidade de um período para outro e a outra não.

Tabela 3

Resultados de testes F para verificar se há diferença nas elasticidades com restrição $\left(\pi^{*}\right)$

\begin{tabular}{l|c|c|c|c|c|c|c|c|c|c|c|c|c}
\hline & $\mathrm{d} .1$ & $\mathrm{~d} .2$ & $\mathrm{~d} .3$ & $\mathrm{~d} .4$ & $\mathrm{~d} .5$ & $\mathrm{~d} .6$ & $\mathrm{~d} .7$ & $\mathrm{~d} .8$ & $\mathrm{~d} .9$ & $\mathrm{~d} .10$ & $\mathrm{~d} .11$ & $\mathrm{~d} .12$ & $\mathrm{~d} .13$ \\
\hline $\mathrm{d} .1$ & - & $\bullet$ & $\bullet$ & $\bullet$ & $*$ & $\bullet$ & $\bullet$ & $\bullet$ & $\bullet$ & $\bullet$ & $\bullet$ & $*$ & $*$ \\
\hline $\mathrm{d} .2$ & & - & $\bullet$ & $\bullet$ & $*$ & $\bullet$ & $\bullet$ & $\bullet$ & $\bullet$ & $\bullet$ & $\bullet$ & $*$ & $*$ \\
\hline $\mathrm{d} .3$ & & & - & $\bullet$ & $*$ & $*$ & $*$ & $*$ & $*$ & $\bullet$ & $\bullet$ & $*$ & $\bullet$ \\
\hline $\mathrm{d} .4$ & & & & - & $*$ & $*$ & $*$ & $\bullet$ & $*$ & $\bullet$ & $\bullet$ & $*$ & $\bullet$ \\
\hline $\mathrm{d} .5$ & & & & & - & $\bullet$ & $\bullet$ & $*$ & $\bullet$ & $*$ & $\bullet$ & $\bullet$ & $\bullet$ \\
\hline $\mathrm{d} .6$ & & & & & & - & $\bullet$ & $\bullet$ & $\bullet$ & $*$ & $*$ & $\bullet$ & $\bullet$ \\
\hline $\mathrm{d} .7$ & & & & & & & - & $\bullet$ & $\bullet$ & $*$ & $\bullet$ & $\bullet$ & $\bullet$ \\
\hline $\mathrm{d} .8$ & & & & & & & & - & $\bullet$ & $\bullet$ & $\bullet$ & $\bullet$ & $\bullet$ \\
\hline $\mathrm{d} .9$ & & & & & & & & & - & $*$ & $\bullet$ & $\bullet$ & $\bullet$ \\
\hline $\mathrm{d} .10$ & & & & & & & & & & - & $\bullet$ & $*$ & $\bullet$ \\
\hline $\mathrm{d} .11$ & & & & & & & & & & & - & $\bullet$ & $\bullet$ \\
\hline $\mathrm{d} .12$ & & & & & & & & & & & & - & $\bullet$ \\
\hline $\mathrm{d} .13$ & & & & & & & & & & & & & - \\
\hline
\end{tabular}

Pela Tabela 3, fica claro que o padrão de mudança das duas elasticidades é o mesmo na maioria dos períodos. Mesmo em períodos que demonstram maior desencontro no padrão de mudança das duas elasticidades (períodos 3 e 5), a quantidade de desencontros é a mesma de encontros, sendo 6 para cada. No total de 78 possíveis pontos de encontros e desencontros no padrão de mudança das duas elasticidades, em 54 deles as elasticidades caminham na mesma direção, 
enquanto em 24 elas possuem caminhos opostos (vão em desencontro), ${ }^{14}$ indicando que existe uma correlação positiva entre as duas elasticidades. Essa evidência corrobora, de um modo geral, o modelo proposto por Thirlwall e Hussain (1982).

Além de termos concluído que as elasticidades mudam através do tempo, é interessante notar que elas tendem a ter um comportamento anticíclico. Podemos ver nas Figuras 1 e 4 que a elasticidade-renda das importações em ambas são maiores em períodos de elevada liquidez externa e de alto crescimento. Isso fica mais evidente quando comparamos os anos 1980 e 1990, pois, em ambas as figuras, notamos uma queda da elasticidade no primeiro período ( $\operatorname{anos} 1980$ ) e uma elevação no segundo (anos 1990). Na Tabela 2, vemos que a queda da elasticidade nos anos 1980 é significativa. Essas evidências indicam que além das elasticidades não serem constantes, elas se alteram de modo a compensar a situação da economia externa, ou seja, quando ela é favorável, as elasticidades tendem a aumentar, caso contrário, elas tendem a diminuir. Portanto, as alterações das elasticidades tendem a amenizar a ocorrência de ciclos econômicos e de mudanças no cenário de liquidez internacional quando comparado com uma situação de elasticidades constantes.

\section{Conclusões}

No presente estudo, através da análise das elasticidades pelo método iterativo dos mínimos quadrados com redistribuição de pesos para corrigir problemas de normalidade dos resíduos, com a adição de variáveis dummies de inclinação, chega-se à conclusão de que as elasticidades, de fato, variam e que o modelo de Thirlwall e Hussain (1982) é compatível em alguns momentos da história da economia brasileira, principalmente entre 1952-1955 e 1980-1983. Em outros, o modelo não mostrou ter um grande poder explicativo, principalmente nos anos 1980 quando o desempenho da economia brasileira foi afetado por crises que atingiram as expectativas dos agentes. Desse modo, mesmo que o crescimento da economia não estivesse restrito pelo desempenho do balanço de pagamentos (BP), os agentes econômicos não estariam com muito ânimo para investimento e consumo, colocando o crescimento da economia aquém do seu potencial dado pelo modelo de Thirlwall e Hussain. Assim, o modelo pode se ajustar bem em grandes períodos de tempo, como alguns estudos indicam, por exemplo Bértola et al.

(14) Se não existisse uma correlação positiva entre $\square$ ' e $\square *$, a quantidade de encontros deveria ser estatisticamente igual ou menor que a quantidade de desencontros. Podemos utilizar o teste de proporções para verificar tal hipótese, ou seja, testar H0: $\mathrm{p} \leq 1 / 2$ contra H1: $\mathrm{p}>1 / 2$, onde $\mathrm{p}=$ número de encontros/(número de encontros + desencontros). Realizando tal teste com um nível de significância de 5\%, encontramos um pc (crítico) igual a 0,59, enquanto dividindo 54 por 78, temos: 54/78 =0,69. Assim rejeitamos a hipótese nula, ou seja, há uma correlação positiva entre as elasticidades. 
(2002) e Jayme Jr. (2001). No entanto, quando períodos menores são analisados, podemos perceber que o modelo se ajusta melhor aos dados.

No trabalho de Nakabashi (2003), é possível observar que as elasticidades-renda das importações variam de modo a compensar o fluxo de capitais, ou seja, quando há períodos de entrada líquida, a elasticidade aumenta e em períodos onde esses recursos são escassos ocorre uma redução da elasticidade, com algo semelhante ocorrendo no presente estudo nos anos 1980 e 1990. Assim, as elasticidades não só variam no tempo, mas servem como um meio de suavizar as restrições externas e, portanto, tornar o processo de crescimento econômico mais estável. Barbosa-Filho (2004) chama a atenção para o fato de que a elasticidade-renda das importações no Brasil é variável e que esse fato é importante na análise das restrições externas, porém ele não faz uma análise mais detalhada dessa questão.

\section{Referências bibliográficas}

ATESOGLU, H. S. Growth and fluctuations in the USA: a demand-oriented explanation. In: SETTERFIELD, M. (Ed.). The economics of demand led growth, challenging the supply-side vision of the long-run. [s.n.t.], 2002. p. 55-63.

A demand-oriented explanation of economic growth in Germany. Journal of Post Keynesian Economics, v. 19, n. 1, p. 101-111, Fall 1996.

BARBOSA, F. H. Crescimento de longo prazo, desindustrialização e política monetária, Brasil x Coréia - 1960: Brasil 3x2 Coréia do Sul, 2000: Coréia do Sul 2x1 Brasil. Boletim Economia \& Tecnologia, Centro de Pesquisas Econômicas (CEPEC) da Universidade Federal do Paraná, ano 2, v. 4, p. 5-8, 2006.

BARBOSA-FILHO, N. H. (2001). The balance of payments constraint: from balanced trade to sustainable debt. New York: Center for Economic Policy Analysis, 2002. p. 1-24. (Working Paper, n. 6).

Growth, exchange rates and trade in Brazil: a structuralist post-Keynesian approach. Nova Economia, v. 14, n. 2, p. 59-86, 2004.

BÉRTOLA, L.; HIGACHI, H.; PORCILE, G. Balance of payments constraint growth in Brazil: a test of Thirlwall's Law, 1890-1973. Journal of Post-Keynesian Economics, v. 25, n. 1, p. 123-140, 2002.

JAYME JR., F.G. Balanced-of-payments constrained economic growth in Brazil. Belo Horizonte: Cedeplar/UFMG, 2001. p. 1-27. (Texto para Discussão, n. 155).

KALDOR, N. The role of effective demand in the short run and the long run. In: BARRÈRE, A. (Ed.). The Foundation of Keynesian Analysis - Proceedings of a Conference held at the University of Paris I - Panthéon-Sorbonne, p. 153-160, 1988.

KRUGMAN, P. Differences in income elasticities and trends in real exchange rates. Cambridge, MA: National Bureau of Economic Research, 1988. p. 1-37. (NBER Working Paper, n. 2761).

Economia e Sociedade, Campinas, v. 16, n. 1 (29), p. 93-110, abr. 2007. 
MCCOMBIE, J. S. L.; ROBERTS, M. The role of the balance of payments in economic growth. In: SETTERFIELD, M. (Ed.). The economics of demand led growth, challenging the supply-side vision of the long-run. [s.n.t.], 2002. p. 87-114.

; THIRLWALL, A. P. Economic growth and the balance-of-payments constraint. Grã-Bretanha: Macmillan Press LTD, 1994.

; Economic growth and the balance of payments revisited. In: ARESTIS, P. PALMA, G.; SAWYER, M. (Ed.). Markets, unemployment and economic policy: essays in honour of Geoffrey Harcout. London: Routledge, 1997. v. 2, p. 498-511.

MADDISON, A. The political economy of poverty, equity, and growth, Brazil and Mexico. Oxford: World Bank, Oxford University Press, 1992. (A World Bank Comparative Study).

MORENO-BRID, J. C. On capital flows and the balance-of-payments-constraint growth model. Journal of Post Keynesian Economics, v. 21, n. 2, p. 283-298, 1998.

NAKABASHI, L. Crescimento com restrição no Brasil: uma abordagem com diferentes elasticidades. Belo Horizonte: Cedeplar/UFMG, 2003. p. 1-18. (Texto para Discussão, n. 203).

OREIRO, J. L. Desenvolvimento numa perspectiva keynesiana: uma resposta ao professor Fernando de Holanda Barbosa. Boletim Economia \& Tecnologia, Centro de Pesquisas Econômicas (CEPEC) da Universidade Federal do Paraná, ano 2, v. 4, p. 9-18, 2006.

PARK, H. M. Testing normality in SAS, STATA, and SPSS. 2003. Disponível em: <http://pytheas.ucs.indiana.edu>. Acesso em 9 ago. 2006.

PORCILE, G.; CURADO, M. Rigidez na balança comercial e movimentos de capital: uma abordagem estruturalista. Revista Brasileira de Economia, v. 56,n. 3, p. 483-495, 2002.

; — _ BAHRY, T. R. Crescimento com restrição no balanço de pagamentos e "fragilidade financeira" no sentido minskyano: uma abordagem macroeconômica para a América Latina. Economia e Sociedade, Campinas, v. 1, n. 1, p. 25-41, 2003.

THIRLWALL, A. P. The balance of payments constraint as an explanation of international growth rate differences. Banca Nazionale del Lavoro Quarterly Review, n. 128, Mar. 1979.

; HUSSAIN, M. N. The balance of payments constraint, capital flows and growth rate differences between developing countries. Oxford Economic Papers, v. 10, p. 498-509, 1982. 


\section{Apêndice}

Nos resultados dos testes, pibd corresponde à taxa real de crescimento do PIB, dpib1 à variável dummy interativa com a taxa de crescimento da renda no primeiro período, dpib2 à variável dummy interativa para o segundo período, e assim por diante. A variável tcambiod representa a taxa de variação da taxa de câmbio real, importacaod a taxa real de crescimento das importações, capitaisd a taxa de variação do saldo da soma das contas de capitais e de serviços, exportacaod a taxa real de crescimento das exportações, dexp1 a variável dummy interativa com a taxa de crescimento das exportações para o primeiro período, dexp2 a variável dummy interativa para o segundo período, e assim por diante.

Resultados dos testes para a regressão por MQO utilizando a equação $m=\varphi j+\pi^{*} y+v D y$.

\begin{tabular}{r|cr} 
Fator de Inflação da Variância & \\
Variable & VIF & \multicolumn{1}{c}{ I/VIF } \\
-0 pibd & 5.21 & 0.191816 \\
dpib7 & 2.97 & 0.337267 \\
dpib6 & 2.83 & 0.353116 \\
dpib3 & 2.21 & 0.452912 \\
dpib2 & 1.91 & 0.522472 \\
dpib4 & 1.82 & 0.548323 \\
dpib8 & 1.77 & 0.564305 \\
dpib9 & 1.66 & 0.601335 \\
dpib10 & 1.61 & 0.619631 \\
tcambiod & 1.45 & 0.690272 \\
dpib12 & 1.42 & 0.705440 \\
dpib11 & 1.36 & 0.737273 \\
dpib5 & 1.29 & 0.777178 \\
dpib13 & 1.15 & 0.866946 \\
Mean VIF & 2.05 &
\end{tabular}

Teste para Heterocedasticidade

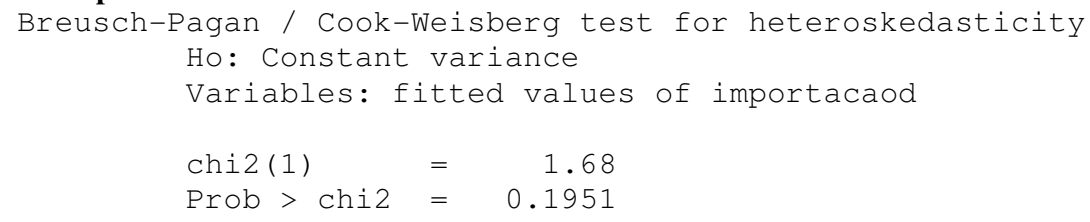

Teste para Autocorrelação dos Resíduos Durbin-Watson d-statistic (15, 53) = 2.388489

Teste para Normalidade

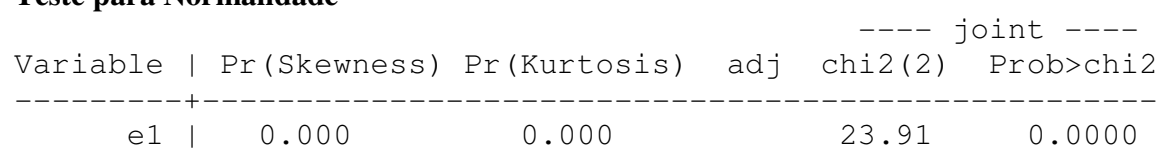

Resultados dos testes da regressão por MQO utilizando a equação $y_{b}=a j+b x+c f_{r}+d D x$ : 
Luciano Nakabashi

\begin{tabular}{|c|c|c|}
\hline \multicolumn{3}{|c|}{ Fator de Inflação da Variância } \\
\hline Variable & VIF & $1 / \mathrm{VIF}$ \\
\hline exportacaod & 10.05 & 0.099493 \\
\hline $\operatorname{dexp} 7$ & 4.54 & 0.220079 \\
\hline dexp11 & 2.09 & 0.478705 \\
\hline dexp10 & 1.98 & 0.505797 \\
\hline dexp2 & 1.87 & 0.535126 \\
\hline dexp9 & 1.78 & 0.562047 \\
\hline $\operatorname{dexp} 4$ & 1.62 & 0.615418 \\
\hline dexp6 & 1.57 & 0.637901 \\
\hline tcambiod & 1.52 & 0.658515 \\
\hline dexp13 & 1.46 & 0.683761 \\
\hline $\operatorname{dexp} 3$ & 1.38 & 0.727004 \\
\hline dexp8 & 1.36 & 0.732887 \\
\hline dexp12 & 1.33 & 0.751101 \\
\hline dexp5 & 1.22 & 0.822755 \\
\hline capitaisd & 1.19 & 0.839011 \\
\hline Mean VIF & 2.33 & \\
\hline
\end{tabular}

Teste para Heterocedasticidade

Breusch-Pagan / Cook-Weisberg test for heteroskedasticity

$$
\begin{aligned}
& \text { Ho: Constant variance } \\
& \text { Variables: fitted values of pibd } \\
& \begin{array}{ll}
\text { chi2(1) } & 1.33 \\
\text { Prob > chi2 }= & 0.2495
\end{array}
\end{aligned}
$$

\begin{tabular}{|c|c|c|c|c|c|}
\hline Variable & $\operatorname{Pr}($ Skewness) & Pr(Kurtosis) & $\operatorname{adj}$ & $\begin{array}{c}----- \\
\operatorname{chi} 2(2)\end{array}$ & $\begin{array}{l}\text { joint }----- \\
\text { Prob>chi2 }\end{array}$ \\
\hline e5 & 0.070 & 0.212 & & 4.81 & 0.0902 \\
\hline
\end{tabular}

Teste para Autocorrelação dos Resíduos

Durbin-Watson d-statistic $(16$, 53) $=1.460155$

Teste para Normalidade 